\title{
UNA ALTERNATIVA EDUCATIVA PARA \\ PREVENIR LA ADICCIÓN AL TRABAJO
}

Mariel Sarai Martínez Dueñas

"Realmente sólo se debe hablar de éxito. El fracaso es un "compañero de viaje" con el que hay que contary se ordena al éxito. Lo importante es tener la actitud adecuada".

Alejandro Armenta

\section{RESUMEN}

Sin lugar a dudas, el contexto del México actual se ha visto influido por un factor muy importante, que es la cultura del éxito profesional. La concepción de "éxito", sobre todo en gente joven, ha cobrado gran relevancia principalmente en el aspecto de la vida profesional. Esta concepción de éxito es un factor determinante en la realización del trabajo, medio humano indispensable para alcanzar la trascendencia.

El objetivo de este escrito es reflexionar en torno al trabajo, ligado directamente al empleo, e influenciado por la cultura del éxito profesional -entendido como poder y riquezas, más que como calidad de vida y actitud de satisfacción-, que ha traído como consecuencia una enfermedad de nuestro tiempo: la adicción al trabajo.

La educación tiene un papel fundamental ante esta problemática social que no ha terminado de proliferar aún en México, al menos de manera consciente. La propuesta de estrategias concretas en torno a la educación para el manejo 
del éxito puede ser una buena alternativa de prevención contra esta adicción.

\section{ABSTRACT}

No doubt, the context of the current México, has been influenced by a very important factor, which is the culture of professional success. The conception of success, mainly among young people, has charged great relevancy principally in the aspect of professional life, being the conception that has of success a determinant factor in the accomplishment of work, which is human indispensable way to reach the transcendency.

The aim of this article is to think around work tied directly to employment and influenced by the culture of professional success, understood as power and wealths, more than as quality of life and attitude of satisfaction, which have brought as consequence a disease of our time: workaholism.

Education has a fundamental role against this social problematic, that still in Mexico has not totally profiler at least in a conscious way, that is to propose concrete strategies concerning education of how to manage success, that can be a good alternative of prevention against this addiction.

Palabras clave : Actividad laboral adictiva; actividad laboral; adicción al estrés laboral; adicción al trabajo; adicción; adicciones psicológicas; adicto al trabajo; autoconcepto; calidad de vida; codependencia; cultura del éxito profesional; dedicación laboral intensa; demandas estresantes del trabajo; distrés; educación para el manejo del éxito; educación; egoísmo; 
empleo; enfermedad; estrés laboral; estrés; estresor; eustrés; éxito profesional; felicidad; fenómeno adaptativo; fracaso; humanidad.; laboro-dependencia; obsesión; patrón A de conducta; pedagogía laboral mexicana; pérdida de tiempo; persona; politoxicomanía; prestigio; prevención; reconocimiento; rendimientos laborales; respuesta de estrés; riesgo de enfermedad; salud física y emocional; secuencia evolutiva; síndrome de estrés; situación estresante; situaciones de estrés; sobreimplicación laboral; tolerancia a la frustración; trabajador adicto e implicado en el trabajo; trabajador adicto y obsesivo; trabajador aislado y solitario; trabajador ambicioso; trabajador competitivo; trabajador culpabilizado; trabajador inseguro; trabajo; trascendencia; trastornos; workabolism.

\section{EL TRABAJO}

El hombre tiene la necesidad de alcanzar la felicidad. Un medio que posee por naturaleza es el trabajo, ante todo el trabajo bien hecho: de manera natural, la persona lo demanda.

Desde el principio, el hombre “... está llamado para el trabajo, pues el trabajo es una de las características que distinguen al hombre del resto de las criaturas, cuya actividad, relacionada con el mantenimiento de la vida, no puede llamarse trabajo; solamente el hombre es capaz de trabajar, solamente él puede llevarlo a cabo, llenando a la vez con el trabajo su existencia sobre la Tierra. De este modo el trabajo lleva en sí un signo particular del hombre y de la humanidad, el signo determina su característica interior y constituye en cierto sentido su misma naturaleza" ${ }^{1}$. Juan Pablo II expresa en la encíclica 
Laborem Exercens, que el trabajo es específicamente humano, pues sólo el hombre decide querer trabajar, esto es en virtud de su humanidad. El trabajo, no es sólo un medio de subsistencia -tal es el caso de las criaturas-, sino que es un medio de trascendencia.

Es necesario puntualizar que el trabajo es más que un contexto social y humano: es una realidad humana innegable.

Es importante además definir qué es. Tomás Melendo, afirma que: "El trabajo podría delinearse como un conjunto de actividades humanas esforzadas, necesarias, con carácter de medio y técnicamente cualificables, por las que los hombres: 1) transforman la naturaleza en beneficio propio, 2) prestan un servicio reconocido a la sociedad, 3) se perfeccionan en cuanto personas" ${ }^{2}$. Esta definición enriquece el concepto de trabajo, considerando elementos como: la transformación del medio, un servicio a la sociedad y el perfeccionamiento propio y común.

Una vez definido lo que es el trabajo y reflexionado en torno al mismo, conviene ahora hacer una diferencia clave para entender la adicción al trabajo y su vinculación con el éxito: distinguir entre trabajo y empleo.

Carlos Armida, afirma que la diferencia que existe entre trabajo y empleo es abismal ${ }^{3}$. El término "empleo" está ligado a un puesto, mientras que el término "trabajo" posee varias 
características, algunas ya mencionadas, que producen cualquier tipo de riqueza y que por tanto enriquecen, no sólo económicamente, sino personalmente a quien lo realiza. El empleo es una forma de trabajar que se originó a principios del siglo XIX, se estructuró como una forma de llevar a cabo las tareas que surgían de la naciente industrialización. Al paso de los años, esta forma de trabajar ha ido penetrando en la sociedad de tal suerte que hoy trabajo y empleo se confunden ${ }^{4}$.

La diferencia crucial se encuentra en que el empleo es sólo una forma de trabajar, mientras que el trabajo es la forma natural que tenemos las personas para desarrollarnos. Al confundir estos dos términos, limitamos los alcances del trabajo, pues se concentra sólo en la búsqueda del dinero. La solución está en dar sentido de trabajo al empleo para encontrar la riqueza cultural, económica, artística, intelectual y espiritual 5 .

Por confusiones de términos, el trabajo se ha reducido al empleo, en gran medida por el contexto actual de la "cultura del éxito" que, entre otras consecuencias, ha generado la adicción al trabajo. Por eso es relevante abordar el tema de la cultura del éxito profesional.

\section{CULTURA DEL ÉXITO PROFESIONAL}

En la actualidad -por lo menos en el México de hoy, y en algunos niveles profesionales y socioculturales-, el éxito profesional se ha convertido en un sinónimo o bien en una aproximación, sumamente cercana, al concepto de felicidad. 
En primer lugar es importante señalar que ante el individualismo de la posmodernidad, el éxito adquiere una connotación eminentemente personal. El éxito, hoy día, es aquel elemento que otorga un grado de valía a las personas. Albert Einstein afirma al respecto: "Trata de no convertirte en un hombre de éxito, sino en un hombre de valor" ${ }^{6}$. Sin embargo, hoy se piensa que la valía personal se adquiere en función del éxito laboral alcanzado. Esto, en términos generales, es un concepto reducido.

Como señalamos, el éxito más importante, según el pensamiento actual, es el alcanzado a nivel laboral: por medio de éste se adquieren prestigio, poder y riquezas. Así, una persona que alcanza el éxito laboral o profesional cree que ha comprado, seguramente, su felicidad.

Según este concepto, el éxito sólo puede ser laboral. Pero, en términos generales, el éxito no puede ni debe ser simplemente laboral, pues el éxito es una actitud ante la vida, que exige unidad en la misma (lo que se conoce como "congruencia de vida"). Para el pensamiento contemporáneo no es comprensible que una persona tenga éxito en su vida laboral, pero se sienta fracasado en su vida afectiva. Esto encuentra su justificación si se entiende que la vida humana no se produce en esferas fragmentadas: la vida posee unidad.

En tanto actitud, la existencia debe reflejar satisfacción personal -que incluye la profesional- ante las actividades realizadas. El trabajo no se reduce al empleo, como ya se mencionó. La vida diaria es trabajo, y es ante lo cual se reacciona con

\footnotetext{
${ }^{6}$ Citado en ARMIDA, C., op. cit., p.12.
} 
aprobación y afecto; es decir, el éxito es una actitud de aprobación, afecto, satisfacción y alegría ante la vida misma y no sólo ante el empleo.

La búsqueda del éxito laboral es una de las primeras causas de la adicción al trabajo, ya que esta actitud exige una "Congruencia" para la que nadie está preparado: disposición total, en todos los ámbitos vitales. Es el éxito el que pretende manejar y dirigir la vida de la persona, cuando debiera ser ésta quien manejara su éxito. Al ser el éxito la consecuencia de una actitud, debiera ser vivido como tal y no el eje manipulador alrededor del cual gira la existencia, situación que -tristemente- se va volviendo más habitual.

El éxito laboral se ha malentendido, olvidando factores como el trabajo bien hecho y la satisfacción personal. En síntesis, el éxito laboral es sólo una división del verdadero éxito. En general, el éxito laboral se traduce en el éxito de la vida profesional; es decir, en la actitud de aceptación y aprobación satisfecha del estilo laboral, por eso si se aprende a manejar el éxito, se aprenderá a trabajar sin convertirse en adicto.

Éste es un esbozo general de uno de los factores contextuales de esta situación. Quedan, pues, aspectos pendientes. Sin embargo es momento de profundizar en la adicción al trabajo como una enfermedad de nuestro tiempo, ello requiere realizar algunas reflexiones educativas en torno al tema, desde el punto de vista preventivo, y vinculadas al manejo del éxito.

\section{LA ADICCIÓN AL TRABAJO}

Los jóvenes mexicanos que empiezan su carrera laboral con éxito, se han concentrado en trabajar. Ramos diría: 
"Trabajar para hoy y para mañana, y no para después" (Ramos, S.; 1998: 59). Esta mentalidad, influenciada por la cultura del éxito profesional, ha generado la adicción al trabajo, además de una serie de factores.

Como ya se mencionó, el trabajo es y ha sido desde siempre una necesidad humana; no obstante, trabajar en exceso y por el simple hecho de trabajar, no personifica sino que esclaviza, perdiendo el trabajo su dimensión ética.

Dentro del trabajo, alcanzar el éxito y la satisfacción laboral son aspiraciones humanas legítimas. Sin embargo el trabajo se torna en adicción, cuando la demanda laboral es tal que "nunca es suficiente para satisfacer" las necesidades. Cuando una persona no maneja adecuadamente su éxito laboral, se adentra en la realidad laboral, convirtiéndola en su única esfera vital y volviéndose así un adicto al trabajo...

A continuación, se analizará la adicción al trabajo desde sus características y generalidades, así como el impacto en la persona, sus consecuencias y alcances.

\section{A. Concepto de adicción}

Antes de analizar específicamente el tema de la adicción al trabajo, es necesario definir el término "adicción" que ha sido, por muchos años, tarea de médicos y otros especialistas dedicados a estudiar adicciones relacionadas únicamente con la ingestión de determinadas sustancias (alcohol, drogas, etcétera). Estas definiciones evidentemente se basan en aspectos relacionados a la salud física y la dependencia frente a alguna sustancia, y aunque consideran otras dimensiones de la persona -como la mental, por ejemplo-, en realidad se refieren a la 
pérdida de la salud "física", como desencadenante de trastornos mentales. En 1935, la Organización Mundial de la Salud aprobó la siguiente definición de alcoholismo, aplicándola posteriormente al término "adicción":

"Enfermedad primaria, crónica, progresiva y mortal con factores genéticos, psicosociales y ambientales que influyen en su desarrollo y manifestaciones. Se caracteriza por presentar en forma periódica o continua: pérdida del control sobre la bebida, preocupación por la sustancia, uso de la misma a pesar de sus adversas consecuencias y distorsiones en el pensamiento, principalmente la negación " 7 .

Debe señalarse que no sólo existe "una" adicción: son tantas como sustancias, situaciones y actitudes haya, ante las cuales las personas pueden aferrarse y crear una relación de co-dependencia.

Enrique Echeburúa afirma que: "Cualquier conducta normal placentera tiende a repetirse y es, por ello, susceptible de convertirse en una conducta adictiva" ${ }^{8}$. Una adicción es, en sí misma, una enfermedad que encuentra su raíz en la relación que se establece con la sustancia adictiva; por eso el ser humano es totalmente responsable de su recuperación, pero no de su etiología.

Definición propuesta por la Asociación Americana de Medicina Adictiva ASAM. En primer lugar fue una propuesta para definir la Enfermedad del Alcoholismo, sin embargo, en 1935, se aprueba como la definición oficial de adicción.

8 ECHEBURÚA, E., ¿Adicciones sin drogas? Las nuevas adicciones: juego, sexo, comida, compras, trabajo, Internet..., p.14. 
Por practicidad científica se han agrupado a las adicciones en dos grandes grupos ${ }^{9}$, como se explica a continuación:

- Adicciones ingestivas: Se caracterizan por la dependencia a ciertas sustancias artificialmente refinadas o producidas que deliberadamente se introducen en el cuerpo ${ }^{10}$ (ejemplo: el alcoholismo).

- Adicciones de proceso: Se caracterizan porque la persona queda atrapada en el mismo proceso, obligada a una serie de acciones o interacciones específicas. En general son formas enfermas o destructivas de adaptarse al mundo ${ }^{11}$ (ejemplo: la adicción al trabajo).

Es esencial señalar que existen componentes fundamentales dentro de una adicción que, según Echeburúa y Corral, son: la falta de control y la dependencia ${ }^{12}$. Es aquí donde encuentra su fundamento la división presentada anteriormente, pues las adicciones no deben limitarse a las conductas generadas por sustancias químicas; existen hábitos aparentemente inofensivos -como es el caso del trabajo-, que pueden convertirse en adictivos e interferir gravemente en la vida diaria de las personas.

Una vez entendido el concepto de adicción y sus diferentes variantes, es momento de aproximarse a la adicción al trabajo. 


\section{B. Definición y generalidades de la adicción al trabajo}

El término "adicción al trabajo" -o workabolism como surge originalmente-, lo propone Oates (1971) en su libro Confessions of a Workaholic. Él define la adicción al trabajo como: "the compulsion or the uncontrollable need to work incessantly" ${ }^{13}$ ("compulsión o necesidad incontrolable de trabajar incansablemente").

La adicción al trabajo, "laboro-dependencia" o workabolism, es una adicción psicológica, emocional o de proceso. Se le considera adicción y no vicio, pues este último es una categoría moral, mientras que la primera constituye un trastorno mental ${ }^{14}$.

Sin lugar a dudas, la dedicación al trabajo ha sido, desde el principio de los tiempos, una conducta socialmente aceptada, indicadora de responsabilidad, efectividad y, sobre todo, de valía personal. Lo anterior encuentra su justificación en que además de ser una fuente de ingresos, el trabajo proporciona a las personas una serie de elementos que influyen en el equilibrio personal ${ }^{15}$. Aunado a lo anterior, una persona que trabaja mucho es admirada; por eso es que la laboro-dependencia, en algún momento, llegó a considerarse como una «adicción positiva” ${ }^{16}$, lo que resulta irónico, pues al hacer referencia a una adicción, necesariamente se engloba como una enfermedad: implica la pérdida de la salud que, evidentemente, no puede ser positiva.

\footnotetext{
3 BUlens, M., "Addicted to work?", en Personnel Today., p.1.

ECHEBURÚA, E., op. cit., p.15.

${ }^{15}$ Ibidem, p.54.

${ }^{16}$ Cfr. FernándeZ-MONTAlvo, J., et al., op. cit., pp.47-49.
} 
Sin embargo, por ser trabajo una conducta socialmente aceptada, el estudio de esta adicción es relativamente reciente. Como ya se mencionó, Oates fue el primero en utilizar el término workaholism ${ }^{17}$, y consideró el proceso del trabajo como un trastorno no grave, del que pueden derivarse varias consecuencias físicas y psicológicas.

Fernández Montalvo y Garrido Landívar, han definido la adicción al trabajo de la siguiente manera:

"La adicción al trabajo se caracteriza por una implicación progresiva, excesiva y desadaptativa a la actividad laboral, con una pérdida de control respecto a los límites del trabajo y una interferencia negativa en la vida cotidiana (relaciones familiares y sociales, tiempo de ocio, estado de salud, etcétera). Al margen de las percepciones distorsionadas del sujeto, la sobre implicación laboral responde al ansia o necesidad de la persona -el trabajo de este modo genera una excitación que oscila entre la fascinación y el sobresalto-, más que a las necesidades objetivas del entorno laboral, ${ }^{18}$.

Según esta definición, la laboro-dependencia -al igual que cualquier otra adicción- es una enfermedad que resulta de una vida desordenada. Sin embargo, hay que enfocarse al hecho de que es un trastorno, sobre todo en la vida emocional; de ahí la gravedad del hecho. Por eso es importante estudiar los rasgos propios de la adicción para detectarla y prevenirla.

\footnotetext{
${ }^{17}$ Ídem.

${ }^{18}$ FERNÁNDEZ-MONTALVO, J., et al., op. cit., pp.49-50.
} 


\section{c. Síntomas, señales de alarma, características y rasgos de la adicción al trabajo}

En definitiva, cuando se hace referencia a la adicción al trabajo, no debe perderse de vista que lo que está en juego es la propia estima y el reconocimiento social ${ }^{19}$, pues tal como puntualiza Enrique Echeburúa: «La dedicación intensa al trabajo se ha considerado durante muchos años como una conducta adecuada y socialmente valorada que denota un sentido elevado de responsabilidad" ${ }^{20}$.

La característica específica de la adicción al trabajo es la de no referirse a un objeto habitual de gratificación directa e inmediata (el trabajo es una actividad que exige un esfuerzo que genera una satisfacción personal, una aprobación social, una remuneración económica u otro tipo de gratificación) ${ }^{21}$. Por lo anterior, se afirma que los principales elementos de placer para el adicto al trabajo son el éxito y el poder adquirido a través del desarrollo profesional.

Como ya se explicó, la adicción al trabajo, en sus generalidades, es una adicción como cualquier otra. Los síntomas que se experimentan son los de cualquier pensamiento adictivo ${ }^{22}$, entre sus rasgos destacan:

- Negociación del problema.

- Distorsiones cognitivas de la realidad.

- Necesidad de control.

- Tolerancia creciente.

19 Ídem.

20 ECHEBURÚA, E., op. cit., p.54.

${ }^{21}$ FERNÁNDEZ-MONTALVO, J., et al., op. cit., p. 53.

22 Cfr. TWERSKI, A., El pensamiento adictivo., pp.11-22. 
- Síntomas de abstinencia (irritabilidad, depresión, ansiedad, etcétera) en los períodos vacacionales (períodos de abstinencia del adicto al trabajo).

Ahora bien, es importante saber que existen "señales de alarma" que el adicto al trabajo presenta antes de convertirlas en rasgos o características vitales. Ante éstas, el potencial adicto al trabajo es capaz de equilibrar su entrega laboral. Cabe señalar que hoy estas señales son socialmente aceptadas, reconocidas e inclusive propiciadas. Ellas son ${ }^{23}$ :

- Prisa constante y ocupación continua. Estar implicados en un mínimo de 2 ó 3 tareas simultáneamente y rechazar actividades.

- Necesidad de control. Rigidez de pensamiento, planear y organizar excesivamente, no delegar el control en otras personas.

- Dificultades en las relaciones personales. Son consideradas como pérdida de tiempo.

- "Embriaguez" de trabajo. Etapas de sobre-implicación en el trabajo, con otras de reducción drástica del nivel de actividad, resultado de un agotamiento patológico y de una falta de descanso (físico e intelectual).

- Dificultad para relajarse y divertirse. El ocio se percibe como una pérdida de tiempo.

- Pérdidas parciales de la memoria. Fruto del agotamiento y de la atención simultánea a muchos asuntos.

- Impaciencia e irritabilidad. El tiempo es una posesión muy preciada, por lo que estas personas carecen de paciencia y se irritan fácilmente ante las pérdidas del tiempo. 
- Déficit de autoestima. La baja autoestima les conduce a la obtención de logros, que aumentan transitoriamente la autoestima.

- Inatención a las necesidades de salud. Son frecuentes una amplia variedad de problemas físicos (obesidad, consumo excesivo de alcohol, tabaquismo, hipertensión...), así como un descuido de las necesidades personales de salud (horas de sueño, descanso, regular, revisiones periódicas, etcétera).

En definitiva, la adicción al trabajo es una adicción que quienes son especialistas en el tema (Killinger, el primero) afirman que se caracteriza por los siguientes rasgos ${ }^{24}$ :

- Implicación elevada en la actividad laboral. El trabajo es, en sentido estricto, su única preocupación, eliminando esferas importantes de su vida.

- Impulso a trabajar debido a presiones personales o internas. El trabajo es una actividad realizada de manera compulsiva y obsesiva, de tal forma que obedece no sólo a las presiones normales que el trabajo en sí mismo refiere, sino que más bien obedece a conductas de exigencia extrema, generadas por la necesidad de construcción de un concepto y sentimiento de estima personal.

- Poca capacidad para disfrutar la tarea realizada. El adicto al trabajo ha perdido la capacidad de gozo y deleite por lo que hace como resultado del estrés y presión interna que le provoca el trabajar; sin embargo, ignora estos sentimientos pues sus necesidades de reconocimiento son más fuertes. 
- Búsqueda de poder y prestigio. El adicto al trabajo, encuentra en éste, el medio para satisfacer sus necesidades de poder y estima, pues le representa una "zona de seguridad" que él cuida exageradamente, por miedo a perderla.

- Aumento excesivo de los rendimientos laborales. Esta característica se refiere a una de las primeras señales de alarma. El hombre en potencia de ser adicto al trabajo, repentinamente y sin dar muestras de preocupación, empieza a "dar más en su trabajo", a trabajar por períodos aún más prolongados y a producir mejores resultados de los que nunca había dado. Así, poco a poco, el sujeto se convierte en un empleado que todo el tiempo trabaja y no hace más que eso; es alguien "ejemplar" al que nadie ve con alarma pues cada día es más productivo. Rasgo característico de este síntoma, es el aumento de la jornada laboral y la evasión del descanso. Generalmente se escuchan frase como: "Hoy no puedo y mañana tampoco", "Tengo mucho trabajo, pero puedo terminar hoy mismo", "Me voy a quedar más tiempo".

- Profundo sentido del deber. Este rasgo, se ve reflejado en actitudes en las que el trabajador siempre hace lo que debe con un estricto rigor, al grado de parecer obsesivo y vigilante del "deber".

- Vida carente de aficiones. Al ser el trabajo su único interés, el adicto pierde repentinamente el sentido de la diversión y el gusto por otros intereses que no sean los estrictamente laborales; por eso, únicamente habla del trabajo y le dedica su tiempo libre.

- Sentimiento de culpabilidad por disfrutar el ocio. Lo percibe como pérdida de tiempo. Recordemos que el tiempo, para cualquier adicto, adquiere una concepción errónea; 
el adicto al trabajo no es la excepción. El tiempo es un recurso no renovable y que adquiere su valía en la cantidad de trabajo productivo que es capaz de generar; así, para un adicto al trabajo, el descanso implica no-productividad. Un adicto al trabajo, sólo es capaz de disfrutar el ocio en el sentido en que éste se vuelve una actividad competitiva ${ }^{25}$.

- Insatisfacción. El adicto al trabajo no conoce la idea de satisfacción pues, de hecho, está a disgusto consigo mismo, lo que hace que no encuentre gusto en ningún tipo de actividad, sentimiento, actitud, etcétera.

- Idea recurrente sobre pérdida de tiempo. La jornada laboral nunca le es suficiente, sus días son más largos y sus noches muy cortas. Pero sobre todo, siempre coquetea con la idea de si lo que realiza será o no una pérdida de tiempo.

Una vez que se comprenden los rasgos de esta adicción es importante explicar ahora cómo surge; es decir, explicar su secuencia evolutiva.

\section{Secuencia evolutiva de la adicción al trabajo}

Antes de estudiarla, conviene hacer referencia al hecho de que, dentro de las adicciones psicológicas, no es frecuente encontrar pacientes que presenten lo que en las adicciones químicas se conoce como "politoxicomanía" ${ }^{26}$. Es decir, no es habitual que, por ejemplo, un adicto al trabajo también sea adicto al juego o adicto a las compras. Sin embargo, debe señalarse que hay una excepción: es muy común que coexistan la

25 Ídem.

26 Presencia de dos o más adicciones químicas en un mismo sujeto. 
adicción al trabajo y la adicción al estrés laboral. Además, resulta usual que un adicto al trabajo presente alguna adicción química -como por ejemplo la adicción a las drogas, muchas veces a las legales-, a fin de neutralizar el agotamiento ocupacional e inclusive para conciliar el sueño ${ }^{27}$. Lo anterior se subraya para entender mejor cómo es que surge y a la par de qué otras enfermedades se origina.

En 1996, Alonso y Fernández ${ }^{28}$, propusieron una secuencia evolutiva frecuente en los adictos al trabajo:

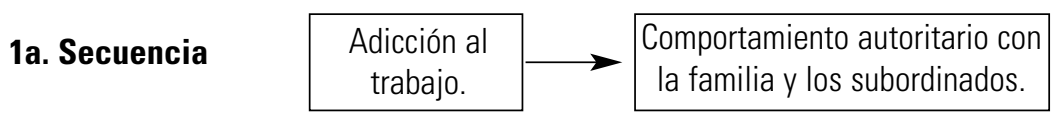

\section{2a. Secuencia}

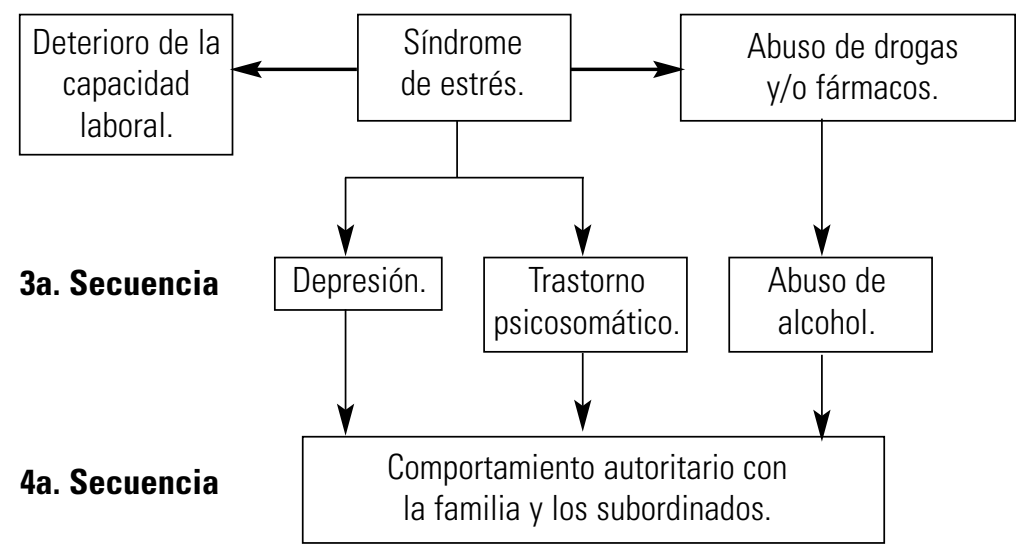

${ }^{27}$ FERNÁNDEZ-MONTALVO, J., et al., op. cit., p.54.

${ }_{28}$ Ídem. 
Como explicación del esquema anterior, se dice que la adicción al trabajo da señales de alerta cuando se observa un comportamiento autoritario con la familia y los subordinados, manifestaciones que alientan el descuido de las relaciones interpersonales. Posteriormente surge el denominado "síndrome de estrés", que desencadena un deterioro de la capacidad dentro del trabajo; por eso, los resultados obtenidos ya no son los óptimos, además se experimenta un abuso de drogas y/o fármacos (por ejemplo, aquellos que facilitan conciliar el sueño, evitan los síntomas de la fatiga, etcétera). Dentro de la tercera secuencia -originada por la segunda-, la adicción al trabajo se manifiesta por medio de depresión, trastorno psicosomático, abuso de alcohol, etcétera; es en esta fase, en la que se presenta la politoxicomanía. En la última fase -donde la adicción al trabajo ya es propiamente una enfermedad-, aparecen nuevamente las manifestaciones caracterizadas por el comportamiento autoritario con la familia y los subordinados.

\section{EL PERFIL DEL ADICTO AL TRABAJO Y SU TIPOLOGÍA}

Para continuar con el análisis, es indispensable realizar una referencia muy precisa a quién es el adicto al trabajo o workabolic. No debe perderse de vista que este sujeto es, en realidad, un adicto común y corriente; sin embargo, en él existen particularidades.

En primer lugar, hay que considerar que el adicto es una persona buena y valiosa que ha llenado huecos de su existencia en formas equivocadas porque no ha podido soportar el dolor interno que le produce la carencia de relaciones sanas y adecuadas ${ }^{29}$. Para sustentar este punto, debe saberse 
que, en muchas ocasiones, las adicciones -y en particular la del trabajo, manifestada en el activismo-, son fugas de la realidad, por las pseudo-concreciones que cada persona hace de la misma.

Se ha caracterizado a quienes presentan una mayor propensión a ser adictos, dentro del Patrón $A^{30}$ de conducta:

- Características psicológicas: Hiperactividad, rapidez, impaciencia, hostilidad, competitividad.

- Relaciones interpersonales: En general son conflictivas con rasgos de dominancia, tensión y agresividad.

- Riesgo de enfermedad: Enfermedad coronaria.

En particular, las personas con Patrón A de conducta y que han desarrollado la adicción al trabajo, pueden ubicarse fácilmente como "ejecutivos agresivos" que tienden a perseguir el control del medio mediante una conducta arrolladora y dominante ${ }^{31}$.

De modo más específico, Aquilino Polaino-Lorente ${ }^{32}$, propone un perfil de adicto al trabajo, del que destacan las siguientes características:

- Piensa en el trabajo cuando no está trabajando.

- No toma vacaciones.

- Es ansioso e inseguro ante las responsabilidades laborales.

- Tiene un compromiso compulsivo y excesivo con la actividad profesional.

- Posee una personalidad obsesiva.

${ }^{30}$ FERNÁNDEZ-MONTALVO, J., et al., op. cit., p.38.

${ }^{31}$ Ibídem, pp.55-56.

32 POLAINO-LORENTE, A., "¿Es usted un workaholic?", en Istmo en línea., p.2. 
- Intenta hacer todo ahora.

- Le es imposible abandonar el trabajo inacabado al final del día.

- Está incapacitado para negarse a nuevas propuestas laborales.

- No tiene un sistema de prioridades estables.

- Es competitivo en cualquier actividad.

- Impaciente.

- Mira frecuentemente el reloj.

- Sus familiares le acusan de estar más interesado en el trabajo que en ellos.

- Se siente culpable cuando no trabaja.

- Espera que todos trabajen como él.

- Posee una mayor iniciativa.

- Manifiesta mayor poder de decisión.

- Se obliga a un aumento de competitividad en el ámbito laboral.

Un típico adicto al trabajo vive con una sobre-implicación laboral, esto sin importar que coexista en él otro rasgo esencial: la adicción al estrés. Actualmente, una competencia laboral importante es el saber trabajar bajo presión; esto se ha confundido con la sobre-implicación laboral, aunada a niveles muy elevados de estrés. Como consecuencia de esta excesiva implicación laboral, se generan problemas de salud física y, sobre todo, el adicto oculta sus pensamientos relacionados con el trabajo para evitar la desaprobación familiar y social ${ }^{33}$ hacia su calidad de vida. Aunque parezca contradictorio, el adicto no expresa su sentir respecto al trabajo para evitar conversar sobre él, ya que se trata de su zona de seguridad y ésta pudiera ser agredida.

33 FERNÁNDEZ-MONTALVO, J., et al., op. cit., p.51. 
Otro rasgo característico, aunque no privativo del adicto al trabajo es la pobre calidad de vida que lleva, generalmente ocasionada por el poco cuidado a su persona, manifestado en: pocas horas de sueño; reducido tiempo libre; ingesta inapropiada y a deshoras de alimentos; algunas veces, consumo abusivo de tabaco y/o alcohol; y en general un ritmo de vida sometido a grandes variaciones.

Naughton (1987) propuso una tipología de adictos al trabajo en relación a dos variables. Por un lado, el grado de compromiso con el trabajo y, por otro, la presencia de rasgos obsesivos ${ }^{34}$. En función de lo anterior, planteó dos tipos de adictos básicos ${ }^{35}$ :

- Trabajador adicto e implicado en el trabajo: Se caracteriza por un elevado grado de compromiso con el trabajo y un bajo nivel de rasgos obsesivos. Por ello, existe una gran probabilidad de desarrollar adecuadamente las demandas laborales. En este caso, la dedicación intensiva al trabajo es un reflejo de la preferencia por las actividades profesionales en relación con otro tipo de intereses.

- Trabajador adicto y obsesivo: Se caracteriza por un elevado grado de compromiso con el trabajo y por la presencia de rasgos obsesivos. Por ello, al resultar una rémora para el rendimiento de este estilo personal (indecisión, perfeccionismo extremo, lentitud, comprobaciones reiteradas, etcétera), la gran cantidad de energía y de tiempo dedicado al trabajo no corresponde con los resultados obtenidos. 
Rohrlich en 1992, clasifica a los adictos al trabajo ${ }^{36}$ según sus hábitos adictivos:

- Trabajador ambicioso: Se trata de una persona habituada a la lucha despiadada para conseguir sus objetivos e imponer sus proyectos en el medio profesional.

- Trabajador competitivo: La persona necesita mostrarse superior a los demás mediante los rendimientos o el esfuerzo.

- Trabajador culpabilizado: La sobrecarga del trabajo se percibe como un imperativo ético, a modo de deformación del mandato bíblico ${ }^{37}$.

- Trabajador inseguro: Busca ansiosamente la aprobación de sus superiores y, con ello, el aumento de los niveles de autoestima.

- Trabajador aislado y solitario: Se trata de una persona desprovista de vínculo sólidos de amistad y de familia. Por eso, las relaciones profesionales y las jornadas de trabajo son un sustitutivo de aquellas relaciones interpersonales gratificantes de las que carece.

Ahora bien, también es conveniente matizar entre la dedicación laboral intensa y la adicción al trabajo, pues no todas las señales son de alerta. Por ello, es indispensable entender esta diferencia, tal como se realiza en el siguiente apartado.

${ }^{36}$ Ibidem, pp.55-57.

${ }^{37}$ Este mandato se refiere a "ganarás el pan con el sudor de tu frente". 


\section{ACTIVIDAD LABORAL INTENSA VS. ACTIVIDAD LABORAL ADICTIVA}

Al estudiar la adicción al trabajo, si se carece de una capacidad para dimensionar el conflicto real, podría caerse en el extremo de considerar todos los hábitos laborales como propensos a ser adictivos. Esto no es así. Es importante establecer una diferencia clara entre las actitudes que implican una actividad laboral intensa y una actividad laboral adictiva.

Generalmente una actividad laboral intensa se caracteriza por estar rodeada de hábitos de trabajo sanos, con las que la persona se personifica, es decir, es capaz de desarrollar habilidades, conocimientos y actitudes propios para la vida, que es el objetivo real del trabajo. Cabe señalar que las personas muy trabajadoras, que aún no son adictas, disfrutan de su trabajo, son altamente productivas y se caracterizan por realizarlo con mucha energía y entusiasmo; además su vida laboral está equilibrada con el tiempo libre y la dedicación a la vida familiar y afectiva. El hábito típico de la persona dedicada al trabajo, más no adicta, es el hecho de saber responder a una demanda objetiva de la sobre-implicación laboral, dándole un carácter de temporal ${ }^{38}$. Mientras que una actividad laboral adictiva transforma los hábitos de trabajo sanos en enfermizos, dándoles un toque de obsesión y co-dependencia, que encuentra sus raíces en la poca o nula autoestima sedimentada en el desempeño profesional.

A continuación a manera de cuadro comparativo ${ }^{39}$, se establecen las diferencias esenciales que proponen Fernández-Montalvo y Garrido:

${ }^{38}$ Cfr. FERNÁNDEZ-MONTAlVO, J., et al., op. cit., pp.50-51.

39 Ídem. 


\begin{tabular}{|l|l|l|}
\hline \multicolumn{1}{|l}{ CONDUCTA } & \multicolumn{1}{l}{$\begin{array}{l}\text { HÁBITOS SANOS } \\
\text { DE TRABAJO }\end{array}$} & \multicolumn{1}{l|}{$\begin{array}{l}\text { ADICCIÓN } \\
\text { AL TRABAJO }\end{array}$} \\
\hline $\begin{array}{l}\text { Dedicación prolongada } \\
\text { al trabajo, incluso en } \\
\text { horas externas al } \\
\text { mismo. }\end{array}$ & $\begin{array}{l}\text { Compromiso. } \\
\text { Implicación. } \\
\text { Dedicación. } \\
\text { Sentido de responsabi- } \\
\text { lidad. } \\
\text { Reconocimiento de las } \\
\text { necesidades puntuales } \\
\text { del trabajo. }\end{array}$ & $\begin{array}{l}\text { Evadir la soledad, el } \\
\text { aburrimiento o el males- } \\
\text { tar personal. } \\
\text { Esfuerzo debido a pensa- } \\
\text { mientos poco realistas. } \\
\text { Incapacidad para equili- } \\
\text { brar la satisfacción deri- } \\
\text { vada del trabajo y la } \\
\text { obtenida a partir de } \\
\text { otros aspectos de la } \\
\text { vida. }\end{array}$ \\
\hline $\begin{array}{l}\text { Alto sentido del } \\
\text { cumplimiento laboral. }\end{array}$ & $\begin{array}{l}\text { Perfeccionista. } \\
\text { Concienzudo. }\end{array}$ & $\begin{array}{l}\text { Intento de justificación } \\
\text { de la excesiva implica- } \\
\text { ción en el trabajo. }\end{array}$ \\
\hline $\begin{array}{l}\text { Control de las activida- } \\
\text { des laborales. }\end{array}$ & $\begin{array}{l}\text { Fiabilidad. } \\
\text { Responsabilidad. }\end{array}$ & $\begin{array}{l}\text { Compensación de la } \\
\text { falta de control en otros } \\
\text { aspectos de la vida. }\end{array}$ \\
\hline Identificación personal. & $\begin{array}{l}\text { Orgullo y satisfacción. } \\
\text { Compromiso con el tra- } \\
\text { bajo. }\end{array}$ & $\begin{array}{l}\text { Utilización del trabajo } \\
\text { para aumentar la auto- } \\
\text { estima que no se obtie- } \\
\text { ne por medio de la } \\
\text { obtención de logros. }\end{array}$ \\
\hline
\end{tabular}

Así, después de analizar los hábitos propuestos en el cuadro anterior, se observa qué difícil es discernir y detectar la adicción al trabajo, ya que en nuestra sociedad, identificada 
como una sociedad de consumo, "prima el éxito y el dinero rápido" ${ }^{40}$. Actualmente se reconocen afirmativamente los hábitos del adicto, dándoles un sentido positivo, pues éstos van de acuerdo con el sacrificio de una vida integral sana en pro del éxito desordenado.

Un rasgo que puede favorecer al reconocimiento de la adicción es el estrés. Ciertamente el "estrés negativo" acompaña el desarrollo de la laboro-dependencia; por tanto, también se desarrolla una adicción al estrés.

\section{EL ESTRÉS LABORAL Y LA ADICCIÓN AL MISMO}

El término estrés, lo propuso Selye en 1926. En sus inicios, hacía referencia a la respuesta general del organismo a una situación estresante ${ }^{41}$. Para efectos de esta investigación, se considera la siguiente puntualización:

“(...) reservaremos el término general de estrés para designar el área o campo de estudio, utilizando el término respuesta de estrés para referirnos a las respuestas inespecíficas del organismo ante cualquier demanda, el de estresor o situación estresante aplicado al estímulo o situación que provoca la respuesta de estrés, y el de efectos o consecuencias del estrés a los efectos producidos en una persona por la emisión de la respuesta de estrés, ${ }^{42}$.

Actualmente, cuando se hace referencia al estrés, se aplica el término a una experiencia negativa que puede llegar a impactar nocivamente nuestra salud. Por ejemplo, se habla del estrés como: desencadenante de ataques al corazón; incremento de los síntomas de la artritis; aumento de la rapidez en

${ }^{41}$ FERNÁNDEZ-MONTALVO, J., et al., op. cit., p.31.

${ }^{42}$ Ídem. 
la multiplicación de las células del VIH, etcétera ${ }^{43}$. Sin embargo, el estrés no necesariamente es una experiencia negativa, pues es un fenómeno adaptativo de los seres humanos que contribuye, en una buena medida, a su supervivencia, a un adecuado rendimiento en sus actividades y a un desempeño eficaz en muchas áreas de la vida ${ }^{44}$. Esta idea, resulta más fácil de comprender si se entiende que el cuerpo humano está biológicamente preparado para responder a las situaciones de emergencia, crisis y tensión, tal y como son las situaciones de estrés.

El cuerpo cuenta con químicos -como la adrenalina y noradrenalina- útiles para responder a las situaciones de estrés ${ }^{45}$ que deberían ser breves. En la actualidad, el ritmo acelerado de vida lleva a vivir constantemente en estrés, y aunque el cuerpo se adapta a estas situaciones, el generar respuestas de estrés en forma excesiva, produce consecuencias negativas en los sistemas de respuesta de las personas; por lo tanto, en función de que la respuesta de estrés sea positiva o negativa (una respuesta positiva puede convertirse en negativa cuando es reiterada), se considera al estrés como eustrés y distrés ${ }^{46}$, respectivamente.

Antes de continuar, debe quedar perfectamente claro que, como ya se mencionó, muchas veces el cuerpo necesita de estrés; de eustrés, para ser concretos, es decir requiere las respuestas positivas de estrés. La adicción al estrés, generalmente al eustrés, se produce cuando la persona se hace adicta a la

\footnotetext{
ALLEN, J., "Stress: An insidious, ubiquitous assassin", en Toronto Star., p.1.

4 FERNÁNDEZ-MONTALVO, J., et al., op. cit., p.31.

45 BARNETCHE, M.E., et al., op. cit., pp.87-88.

46 FERNÁNDEZ-MONTALVO, J., et al., op. cit., pp.32-34.
} 
respuesta de estrés, es decir, a la sensación resultante de las sustancias químicas que responden a dichas situaciones.

Existen generalidades sobre los estresores y para entender bien lo que implica el estrés, debe hablarse de ellos. Los tipos de estresores ${ }^{47}$ se clasifican en función de la respuesta que desencadenan, de la especificidad del momento en que se desencadenan, de la duración, la frecuencia y la intensidad.

En concreto: el estrés laboral involucra a todos aquellos estresores, situaciones y respuestas de estrés relacionados directamente al ambiente laboral de la persona.

Dentro de la adicción al trabajo, el estrés laboral juega un papel esencial. En la mayoría de los casos, un adicto al trabajo presenta su adicción aunada a la adicción al estrés, pues su excesiva implicación laboral, lo ha hecho también habituarse a las situaciones de estrés y el manejo de las mismas. Estas situaciones se caracterizan por estar desencadenadas por un nivel de tensión excesivo, que genera químicos excesivos a los que el cuerpo se habituará y exigirá cada vez en una mayor cantidad. Al igual que le ocurre a un alcohólico con el alcohol conforme su adicción avanza, al adicto al trabajo le sucede con el estrés: su nivel de tolerancia es cada vez mayor por lo que necesita tener más estrés para conseguir la sensación o respuesta que generaba cuando inició su vida laboral, este cuadro se debe a la adicción a la adrenalina que él desarrolla. 
Saber manejar el estrés depende de la forma en que se responda ante el mismo. Por lo anterior, se afirma que un adicto al trabajo generalmente se mueve en el campo del distrés, pues reacciona negativamente ante estas situaciones $y$, evidentemente, los efectos ${ }^{48}$ que vive son negativos.

Para contextualizar el impacto del estrés laboral en un adicto al trabajo, es necesario conocer los seis bloques en que se dividen a los estresores laborales ${ }^{49}$ :

- Estresores del ambiente físico. Ruido excesivo, mala iluminación, temperatura inadecuada, falta de higiene, toxicidad, condiciones climatológicas, inadecuada distribución del espacio físico, falta de espacio físico para trabajar, etcétera.

- Principales demandas estresantes del trabajo. Trabajo por turnos, trabajo nocturno, sobrecarga cuantitativa y cualitativa de trabajo, falta de trabajo, exposición a riesgos y peligros en el trabajo.

- Contenidos de trabajo. Como: el grado en que el ambiente laboral permite al individuo controlar las actividades que realiza, excesivo control de la actividad laboral, excesiva responsabilidad laboral, oportunidad de desarrollo profesional y personal, monotonía de las tareas, ausencia de feedback, falta de identidad de la tarea, falta de identificación del trabajador con la tarea.

\footnotetext{
${ }^{48}$ Los efectos que produce el estrés, se generan en los tres sistemas de respuesta de la persona: fisiológico, cognitivo y motor.

${ }^{49}$ Cfr. FERNÁNDEZ-MONTALVO, J., et al., op. cit., pp.31-34.
} 
- Desempeño de roles, relaciones interpersonales y desarrollo de la carrera. Dentro de estos factores, se agrupan todas aquellas situaciones relacionadas con el puesto dentro de la empresa y grupo de trabajo, así como con el clima laboral y el plan de vida y carrera del trabajador.

- Estresores relacionados con las nuevas tecnologias. Factores directamente relacionados con la incorporación de las nuevas tecnologías al campo laboral y que impactan directamente en la satisfacción laboral. Algunas variables ya identificadas son: el aumento del control sobre el trabajo, la invasión de la privacidad, reducción de contactos sociales, falta de sistemas de apoyo para el aprendizaje y actualización de nuevos sistemas.

- Fuentes extra organizacionales de estrés laboral. Aquí se agrupan factores tales como la familia, tiempo libre, participación en actividades sociales, etcétera.

Con la descripción de estos estresores, puede entenderse cómo cada uno impacta de forma distinta al trabajador, generando así la respuesta de estrés que producirá, a su vez, una cierta respuesta al organismo, a la cual esta persona tiende a ser adicto.

Una vez comprendido todo el contexto de la adicción al trabajo es necesario ofrecer alguna respuesta de prevención. 


\section{¿CÓMO PREVENIR LA ADICCIÓN AL TRABAJO? EL ESQUEMA DE UNA SOLUCIÓN}

Cómo ya se explicó, trabajar es una realidad inevitable y valiosísima, por eso debe aprenderse a realizar esta actividad ordenadamente; es decir, educarse para ser un trabajador íntegro, capaz de actuar con un fin concreto, más allá del éxito.

La "pedagogía laboral" no es más que la sistematización de la educación para el trabajo: se trata de una necesidad primordial del mexicano de hoy y de todos los tiempos. Es una propuesta que surge, en primer lugar, como respuesta a la adicción al trabajo ocasionada por la desvinculación que se vive -o se puede llegar a vivir- entre la vida afectiva y la vida laboral, fruto también del mal manejo del éxito.

Aunque se sabe que a todas las personas se les debe educar para la vida, esta propuesta se dirige principalmente a los jóvenes -etapa de la vida que abarca de los 18 a los 21 añosy a los adultos -etapa evolutiva que abarca de los 22 a los 65 años-, pues es en donde la vida profesional se encuadra de manera más formal, es decir dentro de un empleo, donde las personas tienen mayores posibilidades de presentar la adicción al trabajo, sobre todo por la influencia de la cultura del éxito profesional y el mal manejo del mismo. La pedagogía laboral debe llevarse a cabo por todo tipo de educadores, requiere una interdisciplinariedad entre la familia, los centros educativos y los centros de trabajo.

Para concretar esta propuesta, debe señalarse que agrupa los tipos de educación sugeridos en tres tipos fundamentales y los aspectos que ésta debiera cubrir: 




En concreto, esta reflexión se centrará en la educación para el éxito que se agrupa dentro de la educación para la trascendencia.

\section{UNA REFLEXIÓN ENTORNO A LA EDUCACIÓN PARA EL ÉXITO}

Cuando se habla de éxito, inmediatamente se asocia con ideas positivas como: prestigio, logros alcanzados, respeto, admiración, honorabilidad, alta autoestima, motivación... Ciertamente, al relacionar el éxito con estas ideas, éste resulta muy alentador y atractivo, de tal modo que todas las 
personas desean poseerlo. Sin embargo, existe el lado oscuro del éxito: el fracaso, inclusive hay un miedo muy marcado ante él; de inmediato se le relaciona con la marginación, pobreza, decepción, etcétera, que claramente se entienden como ideas negativas.

Por lo anterior, es preciso contar con caminos concretos para educar para el éxito, herramientas que ciertamente tendrán una intervención positiva en la prevención de la adicción al trabajo. La propuesta es la siguiente:

- Presentar un nuevo concepto de éxito pues en el contexto actual, éste es reduccionista. Por eso debe enseñarse que «el éxito es una actitud que refleja satisfacción personal y profesional ante lo que se realiza".

- Resaltar las actitudes y actividades de éxito - personales y sociales- para reforzarlas positivamente.

- La educación para el éxito abarca dos vertientes. La primera: ordenar adecuadamente el sentido común e identidad del mexicano, basados en el motivo de su existencia: la donación; el mexicano se verá a sí mismo como exitoso en la medida en que sea capaz de darse a los demás. Y la segunda vertiente: consistente en la aceptación objetiva del éxito y el aprender a manejarlo; es decir, cuando dentro de un contexto realista se identifica algo como éxito, hay que educarse a sobrellevarlo de tal suerte que se entienda que la valía personal no es directamente proporcional al grado de éxito alcanzado.

- Manejo adecuado del éxito, ya que actualmente, dentro de la laboro-dependencia, el trabajo es evasión de la falta 
de éxito en otros aspectos de la vida, y al ser el empleo el único lugar de logros, se concentra en él todo lo que se es, evitando así el auto conocimiento.

- La educación para el éxito se inicia desde la formación del auto concepto, pues es muy importante reconocer que la valía personal se posee en tanto que se es persona y en tanto que se actúa conforme a la ética y la moralidad.

- Aprender a darse a sí mismo: entonces se comprenderá que, efectivamente, el éxito es una actitud de satisfacción meramente personal nacida de la donación vivida en los diferentes ámbitos de la vida. Por todo lo anterior, al educar para el éxito debe retomarse la educación en la generosidad y nunca olvidar la dimensión afectiva de la persona.

- Y por último, la formación de la segunda vertiente de este tipo de educación, referida principalmente a la formación para reconocer el éxito y manejarlo adecuadamente. Si se ha concebido el éxito como una actitud de satisfacción personal que nace de la donación, entonces esta fase será más sencilla.

- Aprender a reconocer el éxito, depende del grado de madurez de cada persona, pues en función de ésta, sabrá estar ubicada dentro de su realidad; así, percibirá objetivamente sus logros y sabrá reconocerlos con humildad. Por lo anterior, este tipo de educación también debe procurar la formación de la bumildad: aprender a reconocer los aciertos y limitaciones personales desde la realidad. 
- Tener una sólida formación en lo que respecta a la tolerancia a la frustración: de los errores se obtienen aprendizajes altamente significativos. La tolerancia a la frustración, en gran medida, prepara para el éxito. Fracasar es formativo e inclusive necesario, ya que sólo así se valorará que el éxito es una actitud netamente personal; las expectativas personales que hacen alguna situación exitosa no pueden radicar en un tercero, pues el individuo no lo controla.

Por todas las ideas anteriores, y a manera de síntesis, puede afirmarse que la educación para el éxito se cultiva desde la infancia hasta la tercera edad, sobre todo cuando se permite experimentar el fracaso y sobre todo la donación, fundada en el autoconomiento.

\section{CONCLUSIONES}

- En la actualidad, es evidente que los mexicanos jóvenes, que empiezan su carrera laboral y han alcanzado un éxito significativo, sólo se han concentrado en trabajar para proyectar su futuro en función de medios que han convertido en fines: el trabajo visto como adicción.

- La adicción al trabajo es una enfermedad actual. En México, en concreto, aún no es un problema alarmante, por lo que es esencial favorecer hábitos sanos de trabajo, contrarrestando la cultura del éxito profesional.

- Confundir el éxito laboral con la felicidad, puede desencadenar la adicción al trabajo, entonces ni siquiera puede trabajarse de manera ordenada con fines mucho 
más sublimes, pues todo se considerará a nivel de temporalidad.

- La educación para el manejo del éxito es una asignatura pendiente en el México de hoy. Por lo que esta reflexión surge de manera oportuna y pertinente para su aplicación. 


\section{REFERENCIAS BIBLIOGRÁFICAS}

ARMIDA, C., El trabajo no se pierde... se pierde el empleo., México., Instituto Mexicano de Contadores Públicos., 2001., 150 págs.

BARNETCHE, M. E., et al., Libre de adicciones., Editorial Promexa., México., 2000., 241 págs.

ECHEBURÚA, E., ¿Adicciones sin drogas? Las nuevas adicciones: juego, sexo, comida, compras, trabajo, Internet..., España., Descleé de Brouwer., 2000., 114 págs. FERnÁndeZ-MOnTAlvo, J., et al., Psicopatología laboral., Pamplona., Universidad Pública de Navarra., 1999., 123 págs. GILDER, G., et al., La vertiente humana del trabajo en la empresa., Madrid., Rialp., 1990., 143 págs.

MELENDO, T., La dignidad del trabajo. Madrid., Rialp., 1992., 229 págs.

MILlÁN PUELleS, A., Persona humana y justicia social., México., Minos., 2000., 161 págs.

OTERO, O., La educación para el trabajo., Pamplona., EUNSA., 1989., 167 págs.

TWERSKI, A., El pensamiento adictivo., México., Promexa., 2000., 164 págs.

ALLEN, J., «Stress: An insidious, ubiquitous assassin", en Toronto Star. EBSCO HOST: Newspaper Source., Canadá,, 2004, en:

[http://search.epnet.com/direct.asp?an=6FP1355684143\&db=nfh]. Bulens, M., "Addicted to work?", en Personnel Today., EBSCO HOST: Business Source Premier., 2003., p63, 3p, 1 chart, 3c., en:

[http://search.epnet.com/direct.asp?an=8840347\&db=buh]. 
INTILI, D., "WARNING-workaholic!", en Money., EBSCO HOST: Business Source Premier., Australia., August 2002., p18, 3p., en:

[http://search.epnet.com/direct.asp?an=7333856\&db=buh].

JUAN PABLO II., Carta encíclica Laborem Exercens., Castelgandolfo., 1981, en:

[http://www.vatican.va].

POLAINO-LORENTE, A., “Es usted un workaholic?", en Istmo en línea., Año 37., Número 216., México., 1995, en: [http://www.itsmoenlinea.com.mx/articulos/21605.html]. RAMSEY, R., "A word to workaholics", en Supervision., Vol. 63., Issue 2, p6, 3p., EBSCO HOST: Business Source Premier., Febrero 2002, en:

[http://search.epnet.com/direct.asp?an=5980691\&db=buh].

LERMA, H., La felicidad., Ciclo de Conferencias "Abriendo Camino"., Universidad Panamericana-Campus México., 26 de marzo de 2003 (conferencia).

CÁRDENAS, S., El resentimiento., Curso "Promosapiens"., Centro de Educación Familiar Cedros., México., 13 de diciembre de 2003 (conferencia). 